EESTI NSV TEADUSTE AKADEEMIA TOIMETISED. XII KÖIDE

FOOSIKA-MATEMAATIKA- JA TEHNIKATEADUSTE-SEERIA. 1963, NR. 4

ИЗВЕСТИЯ АКАДЕМИИ НАУК ЭСТОНСКОИ ССР. ТОМ ХІІ

СЕРИЯ ФИЗИКО-МАТЕМАТИЧЕСКИХ И ТЕХНИЧЕСКИХ НАУК. 1963, № 4

\title{
ОБ ОЦЕНКЕ ПОГРЕШНОСТИ МЕТОДА КОНЕЧНЫХ РАЗНОСТЕЙ ПРИ РЕШЕНИИ КРАЕВЫХ ЗАДАЧ ДЛЯ ДИФФЕРЕНЦИАЛЬНЫХ УРАВНЕНИИ ПРОИЗВОЛЬНОГО ПОРЯДКА
}

Р. ЮРГЕНСОН

Для изучения погрешности метода конечных разностей на практике часто применяют метод, предложенный Л. Коллацом [3]. По этому методу погрешности определяются решением системы уравнений для погрешностей, в которой высшие производные заменены их максимальными значениями.

Такой метод строг в случае определенных уравнений второго порядка. При уравнениях более высокого порядка оценки погрешности, в общем, не являются строгими, а вычисления, необходимые для оценки погрешности, - довольно трудоемки, так как, кроме нахождения оценок для высших производных, придется решать систему уравнений нередко с большим числом неизвестных.

Целью настоящей статьи является вывести строгие оценки погрешности, которые были бы применимы для различных разностных методов приближенного решения краевых задач для дифференциальных уравнений произвольного порядка.

Наши исследования основаны на использовании матрицы, в некотором смысле близкой к функции Грина (см., напр., $\left.\left[{ }^{4}\right]\right)$. Эту матрицу мы будем называть дискретной функцией Грина. Ее точное определение дается во втором пункте.

\section{1. Система уравнений для погрешностей}

Рассмотрим дифференциальное уравнение

$$
x^{(n)}+\sum_{k=0}^{n-1} p_{k}(s) x^{(k)}=f(s)
$$

с краевыми или начальными условиями

$$
\begin{gathered}
U_{j}(x) \equiv \sum_{k=0}^{n-1}\left[a_{j k} x^{(k)}(a)+b_{j k} x^{(k)}(b)\right]=c_{j} \\
(j=1,2, \ldots, n),
\end{gathered}
$$

где $p_{k}(s), f(s)$ - непрерывные на отрезке $a \leqslant s \leqslant b$ функции.

Предположим, что краевая задача $\{(1),(2)\}$ имеет единственное решение. 
Для приближенного решения задачи $\{(1),(2)\}$ разобьем отрезок $a \leqslant s \leqslant b$ на $m$ равных частей точками $s_{i}=a+i h$, где $h=\frac{b-a}{m}$. Дифференциальное уравнение (1) рассмотрим в точках $s_{l}$ и заменим в нем производные (в точках $s_{i}$ ) разностными соотношениями, пользуясь формулами численного дифференцирования

$$
x^{(k)}\left(s_{i}\right)=\frac{D_{i}^{(k)}\left(x\left(s_{v}\right)\right)}{h^{k}}-R_{i}^{(k)} \quad(k=0,1, \ldots, n) .
$$

Также заменим разностными соотношениями производные $x^{(k)}(a)$ и $x^{(k)}(b)$ в краевых условиях (2)

$$
\begin{gathered}
x^{(k)}(a)=\frac{D_{a}^{(k)}\left(x\left(s_{v}\right)\right)}{h^{k}}-R_{a}^{(k)} \quad(k=0,1, \ldots, n-1), \\
x^{(k)}(b)=\frac{D_{b}^{(k)}\left(x\left(s_{v}\right)\right)}{h^{k}}-R_{b}^{(k)} \quad(k=0,1, \ldots, n-1) .
\end{gathered}
$$

Здесь

$$
\frac{D_{i}^{(k)}\left(x\left(s_{v}\right)\right)}{h^{k}}, \quad \frac{D_{a}^{(k)}\left(x\left(s_{v}\right)\right)}{h^{k}}, \quad \frac{D_{b}^{(k)}\left(x\left(s_{v}\right)\right)}{h^{k}}
$$

- некоторые линейные выражения относительно $x\left(s_{v}\right)$, аппроксимирующие производные $x^{(k)}(s)$ в точках $s_{l}, a$ и $b$ соответственно, а $R_{l}^{(k)}, R_{a}^{(k)}$, $R_{b}^{(k)}$ - остаточные члены соответствующих формул численного дифференцирования.

$$
\begin{array}{r}
\text { При этом } D_{i}^{(0)}\left(x\left(s_{v}\right)\right)=x_{i}, \quad D_{a}^{(0)}\left(x\left(s_{v}\right)\right)=x_{0}=a, \\
D_{b}^{(0)}\left(x\left(s_{v}\right)\right)=x_{m}=b, \quad R_{i}^{(0)}=R_{a}^{(0)}=R_{b}^{(0)}=0 .
\end{array}
$$

После описанных подстановок получается следующая система линейных алгебраических уравнений

$$
\begin{aligned}
\frac{D_{i}^{(n)}\left(x\left(s_{v}\right)\right)}{h^{n}}+\sum_{k=0}^{n-1} p_{k i} \frac{D_{i}^{(k)}\left(x\left(s_{v}\right)\right)}{h^{k}}=f_{i}+R_{i} \quad(i=\tilde{p}, \tilde{p}+1, \ldots, \tilde{q}) & \\
V_{j}\left(x\left(s_{v}\right)\right) & \equiv \sum_{k=0}^{n-1}\left[a_{j k} \frac{D_{a}^{(k)}\left(x\left(s_{v}\right)\right)}{h^{k}}+b_{j k} \frac{D_{b}^{(k)}\left(x\left(s_{v}\right)\right)}{h^{k}}\right]= \\
& =c_{j}+R_{j}^{(\mathrm{kp})} \quad(j=1,2, \ldots, n),
\end{aligned}
$$

где

$$
\begin{gathered}
p_{k i}=p_{k}\left(s_{i}\right), \quad f_{i}=f\left(s_{i}\right), \quad R_{i}=R_{i}^{(n)}+\sum_{k=1}^{n-1} p_{k i} R_{i}^{(k)} \quad(i=\tilde{p}, \tilde{p}+1, \ldots, \tilde{q}), \\
R_{j}^{(\mathrm{kp})}=\sum_{k=1}^{n-1}\left[a_{i k} R_{a}^{(k)}+b_{j k} R_{b}^{(k)}\right] \quad(j=1,2, \ldots, n) .
\end{gathered}
$$

Натуральные числа $\tilde{p}, \tilde{q}$ и функции $D_{i}^{(k)}, D_{a}^{(k)}, D_{b}^{(k)}$ нужно выбирать таким образом, чтобы число неизвестных и число уравнений в системе $\{(6),(7)\}$ совпадали. Пусть в систему $\{(6),(7)\}$ входят неизвестные * $x\left(s_{p}\right), x\left(s_{p+1}\right), \ldots, x\left(s_{q}\right)$. Тогда должно иметь место равенство

* Отметим, что некоторые $s_{j}$ могут выйти за пределы промежутка $a \leqslant s \leqslant b$. 


$$
q-p+1=n+\tilde{q}-\tilde{p}+1
$$

Решениями системы $\{(6),(7)\}$ являются значения $x\left(s_{i}\right)$ точного решения $x(s)$ задачи $\{(1),(2)\}$ в точках $s_{i}$.

Систему уравнений метода конечных разностей получим, отбросив в системе $\{(6),(7)\}$ члены $R_{i} \quad(i=\tilde{p}, \tilde{p}+1, \ldots, \tilde{q}), \quad$ и $R_{j}^{(\mathrm{kp})} \quad(j=1,2, \ldots, n)$

$$
\begin{gathered}
\frac{D_{i}^{(n)}\left(x_{v}\right)}{h^{n}}+\sum_{k=0}^{n-1} p_{k i} \frac{D_{i}^{(k)}\left(x_{v}\right)}{h^{k}}=f_{i} \quad(i=\tilde{p}, \tilde{p}+1, \ldots, \tilde{q}), \\
V_{i}\left(x_{v}\right)=c_{j} \quad(j=1,2, \ldots, n) .
\end{gathered}
$$

При практическом составлении уравнений (9) для конкретных задач во всех точках, кроме некоторых крайних, используют одни и те же формулы численного дифференцирования. Это значит, что почти во всех точках функции $D_{i}^{(k)}(k=1,2, \ldots, n)$, не зависят от $i$. Пусть $D_{i}^{(k)}$ не зависят от $i$ при $i=\bar{p}, \bar{p}+1, \ldots, \bar{q}(\bar{p} \geqslant \tilde{p}, \bar{q} \leqslant \tilde{q})$. Для этих $i$ обозначим

$$
D_{i}^{(n)}\left(x_{v}\right)=\sum_{j=-v_{1}}^{v_{2}} d_{i} x_{i+j} \quad(i=\bar{p}, \bar{p}+1, \ldots, \bar{q}),
$$

где постоянные $d_{j}, v_{1}, v_{2}$ определены соответствующей формулой численного дифференцирования.

Предположим, что выражения (11) содержат при $i=\bar{p}$ и $i=\bar{q}$ соответственно крайние неизвестные $x_{p}$ и $x_{q}$.

Тогда имеют место равенства

$$
\begin{aligned}
& \bar{p}-v_{1}=p, \\
& \bar{q}+v_{2}=q .
\end{aligned}
$$

Нашей задачей является нахождение оценок для $\varepsilon_{i}=x\left(s_{i}\right)-x_{i}$. Для получения таких оценок составим систему уравнений для погрешностей, вычитая из системы $\{(6),(7)\}$ систему $\{(9),(10)\}$

$$
\begin{gathered}
\frac{D_{i}^{(n)}\left(\varepsilon_{v}\right)}{h^{n}}+\sum_{k=0}^{n-1} p_{k i} \frac{D_{i}^{(k)}\left(\varepsilon_{v}\right)}{h^{k}}=R_{i} \quad(i=\tilde{p}, \tilde{p}+1, \ldots, \tilde{q}), \\
V_{i}\left(\varepsilon_{\vee}\right)=R_{j}^{(\mathrm{kp})} \quad(j=1,2, \ldots, n) .
\end{gathered}
$$

Систему $\{(14)$, (15) $\}$ преобразуем в систему с однородными краевыми условиями Для этого сделаем в упомянутой системе замену переменной

$$
\varepsilon_{i}=u_{i}+t_{i} \quad(i=p, p+1, \ldots, q),
$$

где $t_{i}$ удовлетворяет условням

$$
\sum_{j=-v_{1}}^{v_{2}} d_{j} t_{i+i}=0 \quad(i=\bar{p}, \bar{p}+1, \ldots, \bar{q}),
$$




$$
\begin{aligned}
& \frac{D_{i}^{(n)}\left(t_{\mathrm{v}}\right)}{h^{n}}+\sum_{k=0}^{n-1} p_{k i} \frac{D_{i}^{(k)}\left(t_{\mathrm{v}}\right)}{h^{k}}=R_{i} \\
& (i=\tilde{p}, \tilde{p}+1, \ldots, \bar{p}-1, \bar{q}+1, \ldots, \tilde{q}), \\
& V_{i}\left(t_{v}\right)=R_{j}^{(\mathrm{kp})} \quad(j=1,2, \ldots, n) .
\end{aligned}
$$

При практическом определении элементов $t_{i}$ пишем их в виде

$$
t_{i}=e_{0} z_{0}(i)+e_{1} z_{1}(i)+\ldots+e_{v_{2}+v_{1}-1} z_{v_{2}+v_{1}-1}
$$

где $z_{i}(i) \quad\left(j=0,1, \ldots, v_{2}+v_{1}-1\right)$ являются линейно-независимыми решениями* уравнений (17).

Докажем, что число условий (18) равно числу постоянных $e_{j}$, т. е. равно $v_{2}+v_{1}$.

Число уравнений (18) равно $n+\tilde{q}-\tilde{p}-(\bar{q}-\vec{p})$.

Вычтем из равенства (13) равенство (12)

Отсюда

$$
v_{2}+v_{1}+\bar{q}-\bar{p}=q-p \text {. }
$$

$$
v_{2}+v_{1}=q-p-(\bar{q}-\bar{p}),
$$

нли, учитывая равенство (8),

$$
v_{2}+v_{1}=n+\tilde{q}-\tilde{p}-(\bar{q}-\bar{p}),
$$

что и требовалось доказать.

Итак, для определения постоянных $e_{j}$ получим из системы условий (18) систему уравнений, в которой число неизвестных и число уравнений совпадают. Предположим, что определитель этой системы отличен от нуля.

Вследствие замены переменной (16) система уравнений $\{(14),(15)\}$ заменяется следующей системой

$$
\left.\begin{array}{c}
\frac{\sum_{j=-v_{1}}^{v_{2}} d_{j} u_{i+j}}{h^{n}}+\sum_{k=0}^{n-1} p_{k i} \frac{D_{i}^{(k)}\left(u_{v}\right)}{h^{k}}=R_{i}-\sum_{k=0}^{n-1} p_{k i} \frac{D_{i}^{(k)}\left(t_{\mathrm{v}}\right)}{h^{k}} \\
(i=\bar{p}, \bar{p}+1, \ldots, \bar{q}), \\
\frac{D_{i}^{(n)}\left(u_{v}\right)}{h^{n}}+\sum_{k=0}^{n-1} p_{k i} \frac{D_{i}^{(k)}\left(u_{v}\right)}{h^{k}}=0 \\
(i=\tilde{p}, \tilde{p}+1, \ldots, \bar{p}-1, \bar{q}+1, \ldots, \tilde{q}) \\
V_{j}\left(u_{\nu}\right)=0 \quad(j=1,2, \ldots, n) .
\end{array}\right\}
$$

С помощью дискретной функции Грина эта система преобразуется к виду более подходящему для оценки погрешности.

* Методы решения разностных уравнений такого тнпа изложены, напрнмер, в монографин А. О. Гельфонда [']. 


\section{2. Дискретная функция Грина и ее основное свойство}

Введем понятие дискретной функции Грина.

О пределени е. Дискретной функцией Грина системы

$$
\sum_{j=-v_{1}}^{v_{2}} d_{i} u_{i+j}=0 \quad(i=\bar{p}, \bar{p}+1, \ldots, \bar{q})
$$

при краевых условиях (20) называется $(\bar{q}-\bar{p}+1) \times(q-p+1)$-матрица $G$, элементы $g_{i k}$ которой (как функции от первого индекса) удовлетворяют условиям

$$
\begin{gathered}
\sum_{j=-v_{1}}^{v_{2}} d_{i} g_{i+j, k}= \begin{cases}0 & \text { при } i \neq k, \\
1 & \text { при } i=k\end{cases} \\
(i, k=\bar{p}, \bar{p}+1, \ldots, \bar{q}), \\
\frac{D_{i}^{(n)}\left(g_{v k}\right)}{h^{n}}+\sum_{j=0}^{n-1} p_{j i} \frac{D_{i}^{(j)}\left(g_{v k}\right)}{h^{j}}=0 \\
(i=\tilde{p}, \tilde{p}+1, \ldots, \bar{p}-1, \bar{q}+1, \ldots, \tilde{q} ; k=\bar{p}, \bar{p}+1, \ldots, \bar{q}) \\
V_{i}\left(g_{v k}\right)=0 \quad(j=1,2, \ldots, n ; k=\bar{p}, \bar{p}+1, \ldots, \bar{q}) .
\end{gathered}
$$

Для каждого фиксированного $k(k=\bar{p}, \bar{p}+1, \ldots, \bar{q})$ система $\{(22)$, (23) $\}$ состоит из $n+\tilde{q}-\tilde{p}+1=q-p+1$ условий. Если определитель этой системы отличается от нуля, то существует единственная дискретная функция Грина задачи $\{(21),(20)\}$.

Основное свойство дискретной функции Грина определяется следующей леммой.

Л ем м. Решение и $_{i}$ системь

$$
\frac{1}{h^{n}} \sum_{j=-v_{1}}^{v_{2}} d_{j} u_{i+j}=r_{i} \quad(i=\bar{p}, \bar{p}+1, \ldots, \bar{q}),
$$

где $r_{i}$ - произвольные постоянные, при условиях (20), выражается формулой

$$
u_{i}=h^{n} \sum_{k=\bar{p}}^{\bar{q}} g_{i k} r_{k} \quad(i=p, p+1, \ldots, q) .
$$

Доказ а тельст в о. В силу (23) элементы $u_{i}$ удовлетворяют условиям (20).

Докажем, что $u_{i}$, заданные формулой (25), превращают систему (24) в тождество.

Нмеем

$$
\frac{1}{h^{n}} \sum_{j=-v_{1}}^{v_{2}} d_{i} u_{i+j}=\frac{1}{h^{n}} \sum_{j=-v_{1}}^{v_{2}} d_{j} h^{n} \sum_{k=\bar{p}}^{\bar{q}} g_{i+j, k} r_{k}=\sum_{k=\bar{p}}^{\bar{q}} \sum_{j=-v_{1}}^{v_{z}} d_{j} g_{i+j, k} r_{k} .
$$



зать.

В силу (22) последнее выражение равно $r_{i}$, что и требовалось дока-

Для практического отыскания матрицы $G$ элементы $g_{i k}$ целесообразно писать в виде

$$
g_{i k}=\left\{\begin{array}{c}
a_{0} z_{0}(i)+a_{1} z_{1}(i)+\ldots+a_{v_{2}+v_{1}-1} z_{v_{2}+v_{1}-1}(i) \quad \text { прн } i \leqslant k \\
b_{0} z_{0}(i)+b_{1} z_{1}(i)+\ldots+b_{v_{2}+v_{1}-1} z_{v_{2}+v_{1}-1}(i) \\
(i=p, p+1, \ldots q ; \quad k=\bar{p}, \bar{p}+1, \ldots, \bar{q})
\end{array}\right.
$$

где $z_{j}(i) \quad\left(j=0,1, \ldots, v_{2}+v_{1}-1\right)$ - линейно-независимые решения системы (21).

При таком построении элементов $g_{i k}$ условия (22) будут выполнены для $i=\bar{p}, \bar{p}+1, \ldots, k-v_{2}, k+v_{1}, \ldots, \bar{q}$.

Постоянные $a_{j}, b_{j} \quad\left(j=0,1, \ldots, v_{2}+v_{1}-1\right)$ определяются из условий (22) (при $\left.i=k-v_{2}+1, k-v_{2}+2, \ldots, k+v_{1}-1\right)$ и (23). На основании равенств (8), (12), (13) легко доказать, что число этих условий равно $2\left(v_{2}+v_{1}-1\right)$.

Отметим, что идея введения понятия дискретной функции Грина возннкла при изучении работ И. Шредера [7,8], в которых элементы $g_{i k}$ найдены для задачи

$$
\begin{aligned}
u_{i+1}-2 u_{i}+u_{i-1} & =0 \quad(i=1,2, \ldots, m-1), \\
a_{0} u_{0}-a_{1} l \frac{u_{1}-u_{0}}{h} & =0, \\
b_{0} u_{m}+b_{1} l \frac{u_{m}-u_{m-1}}{h} & =0
\end{aligned}
$$

$(l$ - длина отрезка, на котором рассматривается дифференциальное уравнение; $\left.a_{0}, a_{1}, b_{0}, b_{1} \geqslant 0\right)$.

Для некоторых систем, являющихся системами метода конечных разностей при решении дифференциальных уравнений второго порядка, дискретные функции Грина использованы и другими авторами [5, 6].

\section{3. Оценка погрешности}

Сделаем в системе $\{(19),(20)\}$ замену переменной

$$
\sum_{j=-v_{1}}^{v_{q}} d_{j} u_{i+j}=z_{j} \quad(i=\bar{p}, \bar{p}+1, \ldots, \bar{q}) .
$$

Тогда прн краевых условнях (20), в снлу леммы (из пункта 2),

$$
u_{i}=h^{n} \sum_{k=\bar{p}}^{\bar{q}} g_{i k} z_{k}
$$

и система $\{(19),(20)\}$ прнннмает внд 


$$
\begin{gathered}
z_{i}+\sum_{k=0}^{n-1} p_{k i} \frac{1}{h^{k}} D_{i}^{(k)}\left(h^{n} \sum_{j=\bar{p}}^{\bar{q}} g_{v j} z_{j}\right)=R_{i}-\sum_{k=0}^{n-1} p_{k i} \frac{D_{i}^{(k)}\left(t_{v}\right)}{h^{k}} \\
(i=\bar{p}, \bar{p}+1, \ldots \bar{q}),
\end{gathered}
$$

или, в силу линейности функций $D_{i}^{(k)}$, вид

$$
z=A z+R,
$$

где

$$
\begin{gathered}
z=\left(z_{i}\right), \quad R=\left(R_{i}-\sum_{k=0}^{n-1} p_{k i} \frac{D_{i}^{(k)}\left(t_{\mathrm{v}}\right)}{h^{k}}\right), \\
A z=\left(-\sum_{k=0}^{n-1} p_{k i} h^{n-k} \sum_{j=p}^{\bar{q}} D_{i}^{(k)}\left(g_{v i} z_{j}\right)\right) .
\end{gathered}
$$

Эту систему рассмотрим как операторное уравнение в пространстве $(\bar{q}-\bar{p}+1)$-мерных векторов $R_{\bar{q}-\bar{p}+1}$ и введем норму

$$
\|z\|=\max _{i=\bar{p}, \bar{p}+1, \ldots, \bar{q}}\left|z_{i}\right| .
$$

Обозначим оценки

$$
\begin{aligned}
& \max _{i=\bar{p}, \bar{p}+1, \ldots, \bar{q}}\left|R_{i}-\sum_{k=0}^{n-1} p_{k i} \frac{D_{i}^{(k)}\left(t_{\mathrm{v}}\right)}{h^{k}}\right| \leqslant R^{*}, \\
& \left|t_{i}\right| \leqslant \tau_{i} \quad(i=\bar{p}, \bar{p}+1, \ldots, \bar{q}), \quad \max _{i=\bar{p}, \bar{p}+1, \ldots, \bar{q}}\left|p_{k i}\right| \leqslant \pi_{k}, \\
& \max _{i=\bar{p}, \bar{p}+1, \ldots, \bar{q}} h^{n-k} \sum_{k=\bar{p}}^{\bar{q}}\left|D_{i}^{(k)}\left(g_{v j}\right)\right| \leqslant \mu_{k} \quad(k=0,1, \ldots, n-1) .
\end{aligned}
$$

Легко доказать, что имеют место оценки норм

$$
\|R\| \leqslant R^{*}, \quad\|A\| \leqslant \mu=\sum_{k=0}^{n-1} \pi_{k} \mu_{k} .
$$

Предположим, что $\mu<1$. Тогда на основании теоремы Банаха (см., напр., [2], глава $V, \S 2)$ система $(28)$ имеет единственное решенне $\left\{z_{i}\right\}$ $(i=\bar{p}, \bar{p}+1, \ldots, \bar{q})$, причем справедливо неравенство

$$
\max _{i=\bar{p}, \bar{p}+1, \ldots, \bar{q}}\left|z_{i}\right| \leqslant \frac{R^{*}}{1-\mu} .
$$

Оценки для погрешностей $\varepsilon_{i}$ получим из этого неравенства с помощью соотношений (16) и (27)

$$
\varepsilon_{i}\left|\leqslant \tau_{i}+h^{n} \sum_{k=\bar{p}}^{\bar{q}}\right| g_{i k} \mid \frac{R^{*}}{1-\mu}(i=p, p+1, \ldots, q) .
$$

Правая часть последнего неравенства зависит от дискретной функции Грина и от остаточных членов формул численного дифференцирования $R_{i}^{(k)}, R_{a}^{(k)}, R_{b}^{(k)}$. Члены $R_{i}^{(k)}, R_{a}^{(k)}, R_{b}^{(k)}$, в свою очередь, зависят от 
высших производных решения задачи $\{(1),(2)\}$. Следовательно, точность оценки (29) существенно зависит от того, насколько высшие производные близки к постоянным.

Функция $G$ и элементы $t_{i}$ определяются так, как указано в пп. 1 и 2. Для удобства практического пользования оценкой (29) можно составить таблицы, в которых даются $G, t_{i}$ и оценки $\mu_{k}$ в случае конкретных разностных систем.

\title{
Л И ТЕ Р А Т Р А
}

1. А. О. Ге льфонд, Исчнсление конечных разностей. М., 1959.

2. Л. В. К ан то ров и ч, Г. П. А ки ло в, Функциональный анализ в нормнрованных пространствах. М., 1959.

3. Л. Коллац, Численные методы решения дифференциальных уравнений. М., 1953.

4. М. А. Н а й м а рк, Линейные дифференциальные операторы. М., 1954.

5. А. Н. Тихонов, А. А. С а м а рский, Об однородных разностных схемах, Ж. вычнсл. матем. и матем. физ., 1, 1, 5-63, 1961.

6. M. A. Abdel-Messih, A Green's Function Analogue for Ordinary Linear Difference Equations, Proc. Math. and Phys. Soc. Egypt, 22, 43-51, 1958.

7. J. Schröder, Ober die Differenzenverfahren bei nichtlinearen Randwertaufgaben I, Z. angew. Math. und Mech., 36, 9-10, 319-331, 1956.

8. J, Schröder, Uber die Differenzenverfahren bei nichtlinearen Randwertaufgaben II, Z. angew. Math. und Mech., 36, 11-12, 443-455, 1956.

Ннститут физики и астрононии

Академии наук Эстонской ССР

Поступила в редакцию

$18 . \times 1963$

\section{DIFERENTSIMEETODI VEAHINNANGUST MISTAHES JARRU DIFERENTSIAAL- VORRANDITE RAJAOLESANNETE LAHENDAMISEL}

\author{
R. Jürgenson
}

Resümee

Veahinnangute aluseks on punktis 2 defineeritav diskreetne Greeni funktsioon. Viimase abil teisendatakse vigade vōrrandisüsteem (süsteem $\{(14),(15)\}$ ) kujule, kus veahinnangud on kergesti leitavad.

Punktis 3 toodud ranged veahinnangud on rakendatavad enamiku praktikas kasutatavate diferentsimeetodite korral.

$\begin{array}{cc}\text { Eesti NSV Teaduste Akadeemia } & \text { Saabus toimetusse } \\ \text { Füüsika ja Astronoomia Instituut } & 18 . \text { X } 1963\end{array}$

\section{ON THE ERROR ESTIMATION OF THE FINITE DIFFERENCE METHOD IN SOLVING THE BOUNDARY VALUE PROBLEM OF THE DIFFERENCE EQUATION OF ARBITRARY ORDER}

\section{R. Jürgenson}

\section{Summary}

The error estimation is based on the discrete Green's function, defined in section 2 . This function has been used to transform the system of error equations (section 1) into a form in which the error estimation can be easily carried out.

The strict error estimations given in section 3 are applicable to the majority of the finite difference methods used in practice. 